\title{
Multiple Human Face Detection and Recognition based on LBPH and Machine Learning
}

\author{
Pranjul Kumar $^{1}$, Vashishtha Narayan ${ }^{2}$, Divyanshu chauhan $^{3}$, Astha Singh $^{4}$ \\ ${ }^{1,3,4}$ Department of Computer Science and Engineering, Centre for Advanced Studies, \\ Dr. A.P.J Abdul Kalam Technical University, Lucknow, Uttar pradesh, India. \\ ${ }^{2}$ Department of Computer Science and Engineering, Dr. Shankuntala Misra National Rahabilitation, \\ University, Lucknow, Uttar Pradesh, India \\ Email: kumarpranju1440@gmail.com ${ }^{1}$, vashishthanarayan6446@gmail.com², \\ divyanshuchauhan269@gmail.com ${ }^{3}$, astha0532@gmail.com ${ }^{4}$
}

\begin{abstract}
Face recognition failure is one of the challenging hurdles that a machine deals with. As the revolution of technology comes into the picture, the usability of digital security based on facial biometric become most frequent today. The obsolete security system based on the recognition of features is unable to tackle the variations in a dataset. The failure rate in the face recognition system is found to be common in the various digital systems. This paper introduces a robust design to tackle the recognition issues. This paper utilizes a series of robust techniques in order to establish accurate face recognition with the minimum failure rate. In the proposed design, the facial portion of the acquired image is segmented out using the Haar cascading algorithm which deals with the pixel values of the image. The extracted facial portion of the image is normalized using the normalized pixel intensity algorithm to minimize the noise ratio. Then, the feature extraction procedure is applied to the normalized segmented facial portion of an image which is done by local binary patterns histogram algorithm (LBPH). The face recognition is acquired through such extracted features using the AdaBoost algorithm. The proposed design of face recognition is explored against the testing image with the random variations to generate the accuracy which is found to be $96 \%$ and it is proven better than other existing algorithms.
\end{abstract}

Keywords- Face Detection, Normalize Intensity, Features Extraction, Local Binary Patterns Histogram (LBPH), AdaBoost.

\section{INTRODUCTION}

Detection and recognition of biometric data like facial features for security purposes are very common in today's digital life. With the massive usage, some security risks are also attached such as the failure of a system to locate a person's biometric features that leads to the recognition error. The recognition of facial features is a challenging task in live surveillance in which a system requires to tolerate real-time random variations to identify the images. False rejection and false acceptance rates are the two most common concerns in face recognition. Recognition from the dynamic and variable input image is quite challenging as compared to static input image This paper deals with the recognition challenges of facial data in which multiple variations of a facial image are normalized into a common scale so that the system can able to analyze all the pixels with the same intensity and minimize the recognition fault. The proposed model does face recognition from the dynamic video data which is captured from a video recorder in which each frame contains a sequence of images. The captured images of video frames are used for face recognition in which the proposed model recognizes each identity distinctively. Such a dynamic input frame contains variation in an image in terms of illumination, poses, expression, angles, etc. The chances of recognition failure due to variable exposure of an image increases. The proposed model deals with this challenge and identity each facial data with a minimum error rate and is supportive real-time application like authentication system, forensic investigation, surveillance system, etc.

Face recognition was initially renowned with the introduction of Eigenface features [1] that are uniquely utilized for face recognition. The various face extraction algorithm [2] [3] is also discussed in which Viola-Jones was found most prominent but now it is quite an outdated technique. Recognition of a facial image requires a model for training and testing the facial features. A classification algorithm [3] [4] requires to distinguish a number of facial images using multiple classes. Some earlier techniques like sparse [11], manifold [5] and linear subspace [5] utilized for face recognition that got out-dated. An attention-based design for detecting the data from video input was coined by Yang et al. [6]. Face recognition based on thresholding technique from the video input was also discussed by Sivic [7] et al. A recognition modeling based on an instance of a model was also used earlier. An attention-based face recognition model using deep reinforcement learning is one of the prominent techniques used earlier that was coined by Y. Rao et al. [8]. Such earlier face recognition models may not provide recognition consistency in a huge dynamic dataset in which images may contain a variation of lighting, poses, expressions, etc. The recognition of facial images from a low-quality frame is also a challenging task for such earlier discussed methods.

This main contribution of the paper deals with the dynamic real-time data in which people with various expressions, poses, etc. are captured in video frames. This paper performs the analyses of the captured facial data under multiple noises and disturbances. Some video frames are low-quality frames containing disturbing images. Recognition rate may compromise when it comes to read the facial data from a lowquality frame. The extraction of the facial images from the frame is practiced by using Haar cascade algorithm which basically locates the haar feature on the image to identify the facial portion from the entire image. After, the detected face image is normalized in which intensity of brightness of the pixel values is suppressed or enhanced at the average level. This normalized facial image is enrolled for feature extraction 
process in which hog features, which contains the local and global information of the key points on facial portion, are extracted using LBPH (local binary pattern histogram) algorithm. The recognition and classification part is executed by Adaboost algorithm which applies distance metric to find the distance between the extracted hog features of two facial images in order to check the similarity. The accuracy of recognition is found to be $96 \%$ which, increased than as compare to earlier algorithm, after applying image normalization technique. Usually, the face recognition process comprises four different system: the information gathering system, the highlight retrieval system, the characterization system and the preparation of the database classifier module [11]. The image data gathered from training gathering system and is used as a study test. The classifier prepared by the database which is used in the model to classify tests to determine people's identity.

The paper includes different modules. Module II deals with the methodology proposed. Module III deals with experimental data. Section IV captures the conclusion. The final modules include all the references.

\section{PROPOSED TECHNIQUE}

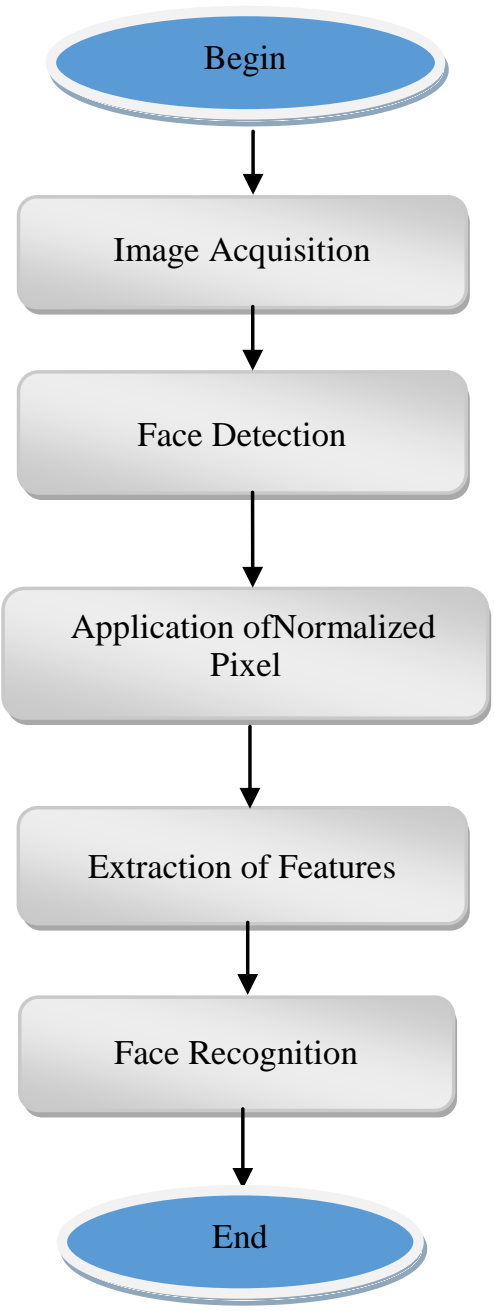

Fig.1: Flowchart of Multiple Face Recognition System

\section{Face Detection}

The OpenCV [5] is used for implementing the haar cascade algorithm in which facial portion is extracted using the haar features that are present on eyes, nose, mouths, etc. This facial information is already learned by a haar cascade classifier based on which it locates the rectangular block over the facial portion of the given image. This method is proposed by Paula voila and Michael jones originally [12]. The essential features of the haar cascade classifier are the haar features, for the detection of the face. The classifier is pre-trained with the set of images and learns their respective facial information. Result of a function in a unit amount is given Sum rectangle blackSum rectangle white: Haar-like characteristics are the features of the rectangle for easy face detection. Some haar-like feature is shown in fig 1. Each feature points defines as the difference of the sum of pixels of white rectangle and the sum of pixels of black rectangle.

\section{$\mathrm{P}(\mathrm{x})=$ Sum rectangle black-Sum rectangle white}
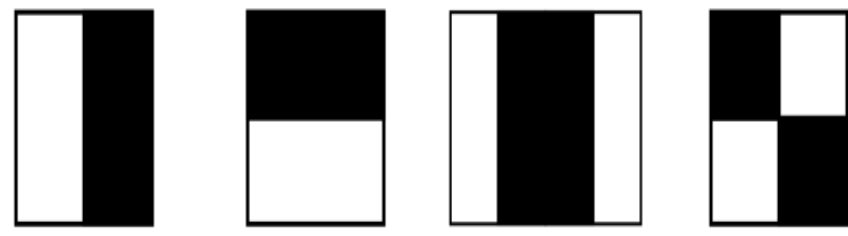

$$
\text { (1) }
$$
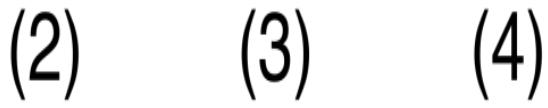

Fig1: Haar features image

\section{Image Normalization}

Normalization technique was applied to all extracted facial images in order to eliminate the disturbance in image(noise) and to align the image.

- Cut the face

- Normalize pixel intensity

- Resize Face Image

Normalize Pixel Intensity is applied before appointing the faces for training the model. The complete grayscale spectrum is used [0-255] in which the Contrast of low-quality pixels is enhanced. The distance between two pixels is extended and consecutively the contrast increases that make the feature points of the face much better. The intensity of the pixel values of an extracted image is made constant throughout the image by using a scaling factor which sets the intensity values to average and applies it to all the pixels. Basically, this method enhances the low bright portion of the image. The normalization of image pixels is done in grayscale. The objective of pixel normalization is to maintain the rank of intensity values without suffering any loss of information. Pixel normalization reduces the error in recognition since all the pixel intensity set to the common average value in which each dull pixel can help in the recognition. In this way, the challenge of recognition from the low-quality frame [10] can be resolved. 


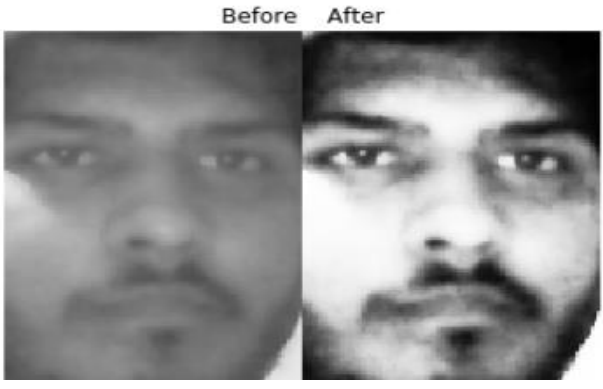

Fig 4: Appling Normalize Pixel Intensity

\section{Features Extraction}

Few analyses have studied that focuses on the retrieval of confined characteristics from images. We don't want to see the high-dimensional matrix in place of the entire picture; we want to define an object's confined characteristics. The characteristics you derive this way would indirectly have a low dimensionality. The image representation given to us doesn't just suffer from variations in illumination. Consider things like size, translation, and rotation in pictures-at least a little resilient against those issues that must reside in the local definition. The technique of Local Binary Patterns [13] is rooted in $2 \mathrm{D}$ pattern determination. The elemental idea behind the Local Binary Patterns is, to sum up, the confined design of an image structure in an image by analyzing the pixels to their neighborhood. Select a pixel value as your middle \& threshold across your neighbors. If a Neighbor's central pixel frequency $>=0$, then it is one, otherwise zero. For each pixel, you'll get a Binary number, just like 11010101 . After this you get (28) feasible sequences with 8 surrounding pixels, is knows as Local Binary Patterns /LBP codes.

$(x)=\left\{\begin{array}{l}1, x \geq 0 \\ 0, x<0\end{array}\right.$

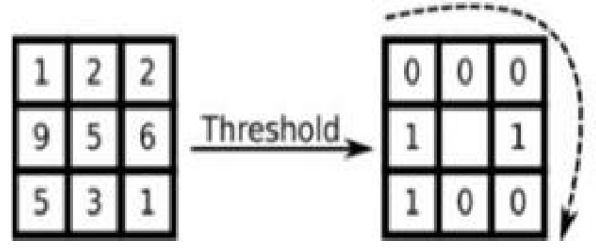

Binary: 00010011 Decimal: 19

Fig 2: Image of LBP Operator

To obtain the feature vectors, the form of a particular pixel is attained. To express all faces proficiently, the image has to be split into G2 parts. The histogram of the particular potential label is composed. In a histogram, every bin gave the information about histogram design thus the feature vectors can be attained from the histogram.By this particular part of histogram contains of $\mathrm{Y}(\mathrm{Y}-1)+3$ bins $\mathrm{Y}(\mathrm{Y}-1)$. With the use of LBP, we attain the area with a distance from the edges of the images. The border of the image is not of any use, if we don't use LBP. With the help of calculating the LBP code for all pixels $(\mathrm{Pd}, \mathrm{Qd})$ with $\mathrm{Pd} \epsilon\{\mathrm{Z}+1, \ldots, \mathrm{M}-\mathrm{Z}\}$ and $\mathrm{Qd} \epsilon\{\mathrm{Z}+$ $1, \ldots, N-Z\}$. If an image is divided into $s \times s$ regions, then the histogram for region $\left(S_{p}, S q\right)$, with $S_{p} \in\{1, \ldots, S\}$ and

$\mathrm{Sq} \in\{1, \ldots, \mathrm{S}\}$,
Algebraically,

$$
\begin{gathered}
L_{i}\left(S_{p}, S q\right)=\sum p, Q J\{L B P X, Y(a, b)=T(j)\}, j=1, \ldots \ldots X(X-1)+3 \\
a \in\left\{\begin{array}{c}
\left\{Z+1 \ldots \ldots ., \frac{M}{S}\right\} S_{a}=1 \\
\left\{S_{a}-1\left(\frac{M}{S}\right)\right\}+1 \ldots \ldots . M-Z \\
\left\{S_{a}-1\left(\frac{M}{S}\right)\right\}+1 \ldots \ldots S_{a}\left(\frac{M}{S}\right)
\end{array}\right\} \text { where } \mathrm{Sa}=\mathrm{S} \\
\text { else }
\end{gathered}
$$

T denotes the Binary $\mathrm{j}$ label and

$$
\mathrm{J}(\mathrm{X})=\left\{\begin{array}{l} 
\\
1, X=\text { True } \\
0, X=\text { False }
\end{array}\right\}
$$

The 2 different stages of the face can be obtained via feature vector: The tag consists of knowledge at the small natural level \& the model of histogram administers the knowledge regarding the face.

\section{Dataset used:}

The database we created stores 300 pictures of each individual. Based on face detection, it is developed. To form the created face database, the saved images are stored in the same directory. The images are converted to gray scale from the dataset images for the feature extraction during the image acquisition process and after that applying normalized intensity on those image's pixels for better identification outcomes. The samples of images from the database are shown in Fig.1.

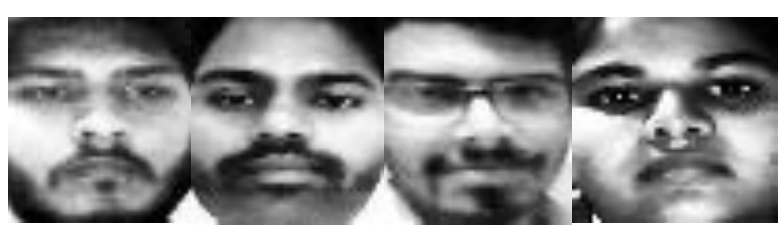

Fig. 3: People's Dataset images

\section{Face recognition:}

The AdaBoost is a classifier, based on machine learning, is used to classify the facial images after the recognition through their features. These features are the local features that are fetched by the LBPH algorithm. The model is trained using the features of training images. The algorithm builds the decision tree for every feature. Then these features are classified using the decision tree. Each class contains the features of a single belonging person. In this way, multiple classes are generated and the model trained themselves according to training features which use for recognition prediction for a new test facial image. AdaBoost [15] is an adaptive boosting algorithm 
in which the model performs the binary classification in which the model gets trained from a weak classifier to a strong classifier based on the features it reads. Each learning state or strong classifier is combined with all the previously learned states. Every iteration of the AdaBoost algorithm minimizes the error in the training set and finally generates the strong classifier. The basic algorithm of AdaBoost is given below:

\section{Initialize the facial images and assign similar weights to each image. \\ 2. Provide the extracted local features randomly as an input to the model and the model is learned with the features identify the errors in learning. \\ 3. The next state of learning will include other features and try to minimize the error in the previous state. \\ 4. In this way, a weak classifier turns into a strong classifier. \\ 5. if (accuracy is achieved with minimum error) \\ Go to step 6 \\ else \\ Go to step 2 \\ 6. \\ End}

The features of the test samples of the facial images are enrolled into the trained model and then the model is able to classify the new features and recognize their belonging class.

\section{Algorithm to recognize face}

Step1: Begin with interim=0

Step2: T is used for train image

Step3: $\mathrm{z}=0$; initialization of pattern histogram.

Step4: calculate normalize pixel intensity.

Step5: Iterate the value of bin by value 1.

Step6: Obtain the largest feature of LBP all along every face image and then append it into the individual vector.

Step7: comparison of features.

Step8: Input is contrasted with the saved database the image is identified.

$$
\operatorname{LBP}\left(P_{d}, Q_{d}\right)=\sum_{N=0}^{7} T\left(j_{n}-j_{a}\right) 2^{t}
$$

Where, $j_{a}$ denotes the central pixel's value, $\left(P_{d}, Q_{d}\right)$ denotes the pixel's information of 8 surrounding.

The model compared the similarity between the test features and the trained features using distance metric and finds the Euclidean distance between the values of the features and finally compares it with a threshold value. The threshold value is changed to increase accuracy. The calculated distance must be smaller than the threshold value in order to prove similarity. Thus, the recognition of facial features is done based on their similarity features with respect to the particular class. Each class separately belongs to an individual identity which signifies the true belongingness.

\section{EXPERIMENTAL RESULTS}

The database is designed by us in which 300 pictures of peoples have taken and stored. Every image in the database has a different number of identities and labels. Begin with preparing the multiple images of the database, the image's LBP texture features are extracted, and then perform intensity normalization [15] on each image. And eventually, the face information is classified and recognized. We have obtained 300 face images of each person for this study.

Then we will compare the extracting features of the input image with the features of dataset images which is then we will figure out that if input image features are successfully matched with dataset image features then the person is recognized otherwise it will not recognize the person and print unidentified face.

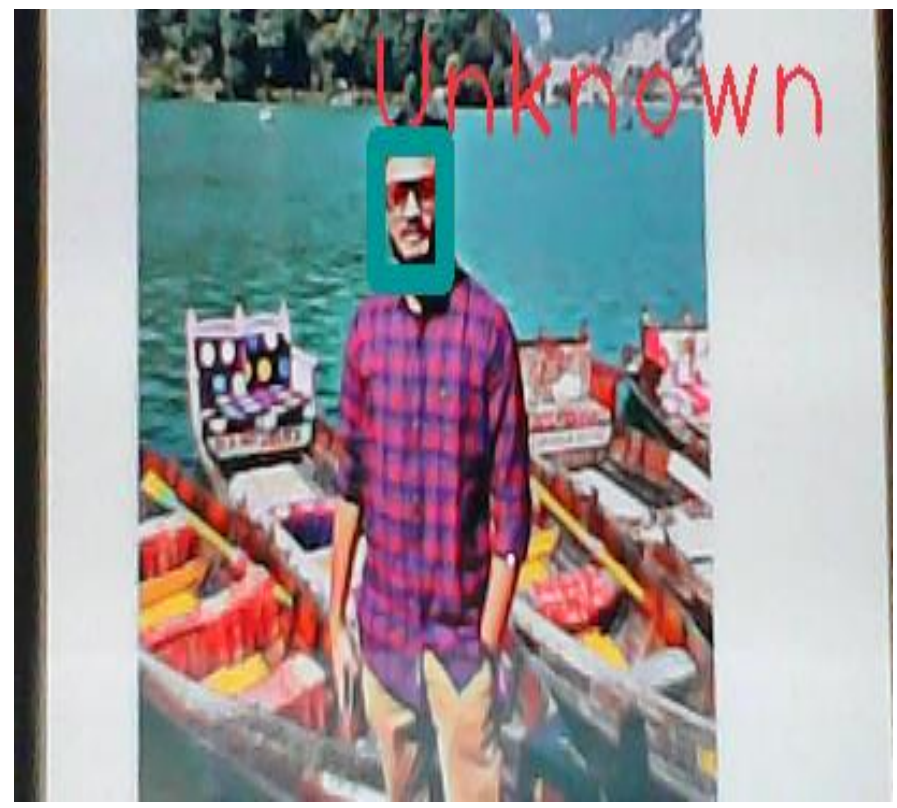

Fig 5: unidentified face

Fig.5. shows an image from test set which is unknown. Its facial portion is extracted and LBPH features are fetched out which is compared with the trained features to know the identity class of the input person's image. Based on the proposed method, features of a facial image of identified (trained) and unidentified person is contrasted with the facial picture of identified persons from the database.

This paper contains some main things-

- Detect the face

- Applying pixel normalization

- Features extraction

- Face recognition

\section{A. Detect the face}

Faces will be detected and cut from the live video via a camera in a grayscale image. HaarCascade classifier is used for the detection of the face. 


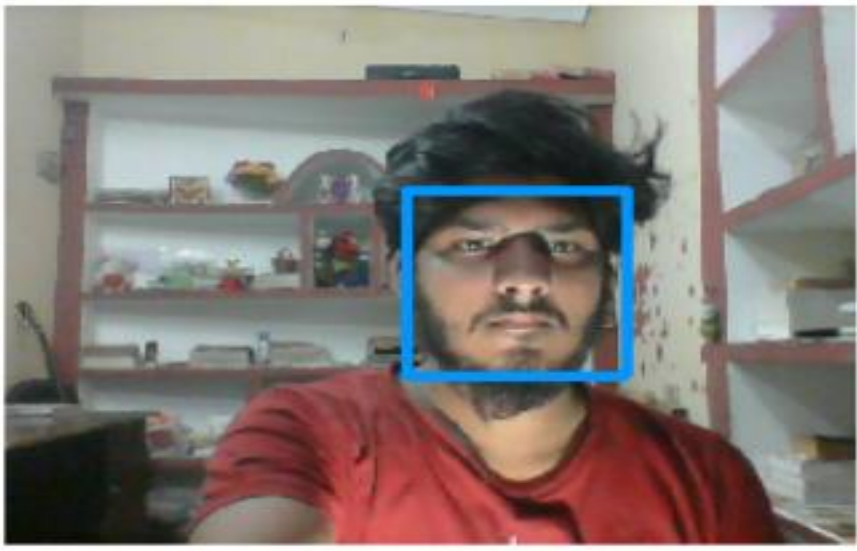

Fig 6: Face Detection

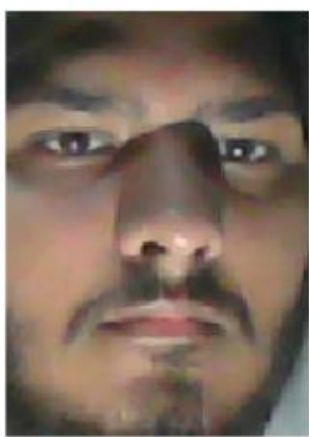

Fig 7:Cut Down the main region from image where features processing is performed

\section{B. Applying pixel normalization}

In this part we normalize the pixel intensity of an image to reduce the noise.

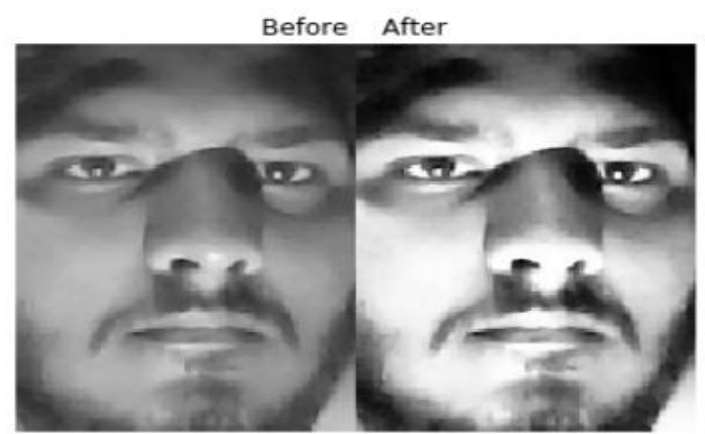

Fig 8: Applying pixel normalization in face image

\section{Recognize Face Image}

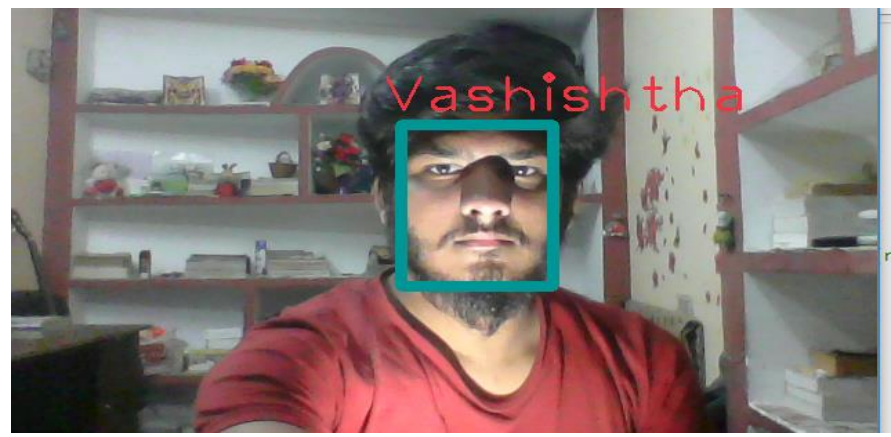

Fig 9: Identification of face in an image

Recognizing facial images is the final task. The LBPH local features of the Face are used for training which is used to identify the unknown LBPH features. The categorizer compares recorded images of the face with images of the face of the output. If the facial characteristic of the inserted image matches with images of the database, then the recognition result will be shown on the output screen.

$\mathrm{S}$

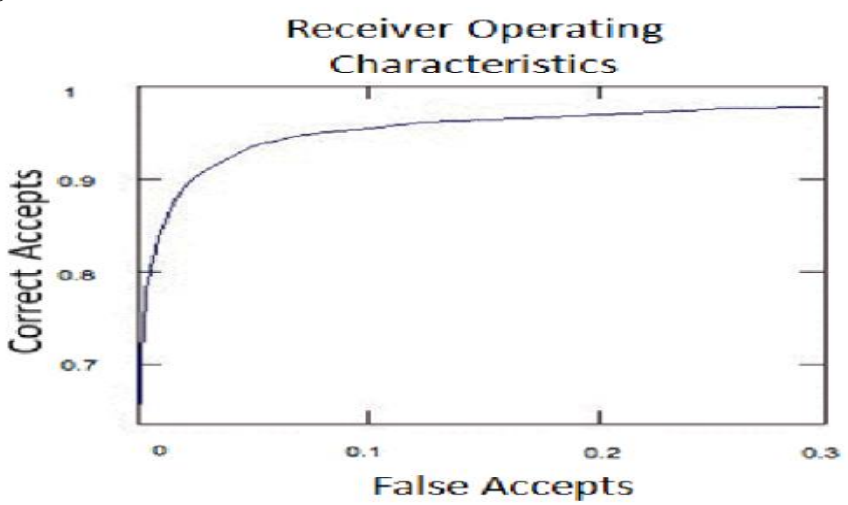

Fig.10. ROC curve for face recognition

Fig.10. shows the accuracy of the matching of test images with the trained dataset. A number of 100 images contained genuine and fake user's picture is used as test samples which are compared with the trained features. The error rate in the recognition is $4 \%$ only which can be improved by changing the threshold value.

Table 1. IDENTIFICATION PRECISION COMPARISON

\begin{tabular}{|l|l|l|}
\hline Face & $\begin{array}{l}\text { Without } \\
\text { nocognition } \\
\text { system }\end{array}$ & $\begin{array}{l}\text { With } \\
\text { process } \\
\text { process }\end{array}$ \\
\hline Accuracy & $93 \%$ & $96 \%$ \\
\hline
\end{tabular}

Table 1 shows the comparision of the recognition accuracy with and without normalization process.

Table 2. Comparison table

\begin{tabular}{clc}
\hline $\begin{array}{c}\text { S. } \\
\text { No. }\end{array}$ & \multicolumn{1}{c}{ Techniques } & Accuracy/Result \\
\hline $\mathbf{1}$ & $\begin{array}{l}\text { Face recognition based on } \\
\text { Rough Contour and } \\
\text { Estimation Routine[11] }\end{array}$ & $92.1 \%$. \\
\cline { 2 - 3 } $\mathbf{2}$ & $\begin{array}{l}\text { Face recognition based on } \\
\text { principle component } \\
\text { analysis[12] }\end{array}$ & $83 \%$ \\
\cline { 2 - 3 } $\mathbf{3}$ & $\begin{array}{l}\text { Face recognition based on } \\
\text { AdaBoost and advance } \\
\text { component analysis.[13] }\end{array}$ & $95.20 \%$ \\
\cline { 2 - 3 } $\mathbf{4}$ & $\begin{array}{l}\text { Radial Symmetry } \\
\text { Transform based face } \\
\text { recognition [14] }\end{array}$ & $83 \%$ \\
& & \\
\hline
\end{tabular}

5

Recognition based on SVM

$95.71 \%$

[15]
$96 \%$. 


\section{Conclusion}

In this paper, the LBPH and Normalize Pixel Intensity Process are used mainly to identify and de-noise the facial features of a particular person. The experiment is done in two ways, first is with the normalization process and the other is without applying the normalization process. So for this, we have collected the results of the two tests, that we had performed on the multiple images $\&$ from the respective tests, we have seen that, the face recognition gives the best results when we applied the Normalize Pixel Intensity Process on the images because it enhances the Pixel Intensity of an image and we get overall accuracy of $96 \%$ of recognition but we also found the less precise accuracy of $93 \%$ of face recognition when we didn't apply the Normalize Pixel Intensity process. And also the Adaboost algorithm is successfully implemented for the training purpose which builds strong classifier from the combination of weak classifiers in order to classify the trained image accurately which help us to recognize the unknown test features.

\section{REFERENCES}

[1] Belhumeur, P. N., Hespanha, J. P., \& Kriegman, D. J. (1997). Eigenfaces vs. Fisherfaces: recognition using class specific linear projection. IEEE Transactions on Pattern Analysis and Machine Intelligence, 19(7), 711720. doi:10.1109/34.598228.

[2] Hwang, J. J., Kim, Y. M., \& Rhee, K. H. (2018). Faces Recognition Using HAARCASCADE, LBPH, HOG and Linear SVM Object Detector. Proceedings of the Sixth International Conference on Green and Human Information Technology, 232236. doi:10.1007/978-981-13-0311-1_39.

[3] Jain, S., Kumar, B. L. S., \& Shettigar, R. (2017). Comparative study on SIFT and SURF face feature descriptors. 2017 International Conference on Inventive Communication and Computational Technologies

(ICICCT). doi:10.1109/icicct.2017.7975187.

[4] Kshirsagar, V. P., Baviskar, M. R., \& Gaikwad, M. E. (2011). Face recognition using Eigenfaces. 2011 3rd International Conference on Computer Research and Development. doi:10.1109/iccrd.2011.5764137.

[5] Xianghua Fan, Fuyou Zhang, Haixia Wang, \& Xiao Lu. (2012). The system of face detection based on OpenCV. 2012 24th Chinese Control and Decision Conference $n$ b(CCDC). doi:10.1109/ccdc.2012.6242980.

[6] LU, W., \& YANG, M. (2019). Face Detection Based on ViolaJones Algorithm Applying Composite Features. 2019 International Conference on Robots \& Intelligent System (ICRIS). doi:10.1109/icris.2019.00029.

[7] J. Sivic, M. Everingham, and A. Zisserman. who are you?learning person specific classifiers from video. In CVPR, pages 1145-1152, 2009.

[8] Y. Rao, J. Lu, and J. Zhou, "Attention-aware deep reinforcement learning for video face recognition," in Proceedings of the IEEE Conference on Computer Vision and Pattern Recognition, 2017, pp. 3931-3940.

[9] Ahmed, A., Guo, J., Ali, F., Deeba, F., \& Ahmed, A. (2018). LBPH based improved face recognition at low resolution. 2018 International Conference on Artificial Intelligence and Big Data (ICAIBD), doi:10.1109/icaibd.2018.8396183.

[10] A. Dwivedi, A. Kumar, M. K. Dutta, R. Burget and V. Myska, "An Efficient and Robust Zero-Bit Watermarking Technique for Biometric Image Protection," 2019 42nd International Conference on Telecommunications and Signal Processing (TSP), Budapest, Hungary, 2019, pp. 236-240.

[11] Jyh-Yeong Chang and Jia-Lin Chen, "Automated Facial Expression Recognition System Using Neural Networks"
Journal of the Chinese Institute of Engineers, Vol. 24, No. 3, pp. 345-356(2001).

[12] G.R.S Murthy R.S.Jadon "Effectiveness of Eigenspaces for Facial Expressions Recognition" International Journal of Computer Theory and Engineering, Vol. 1, No. 5, December, 2009, pp.1793-8201.

[13] Z. Lei, C. Wang, Q. Wang and Y. Huang, "Real-Time Face Detection and Recognition for Video Surveillance Applications," 2009 WRI World Congress on Computer Science and Information Engineering, Los Angeles, CA, 2009, pp. 168172.

[14] NeetaSarode,Prof.ShaliniBhatia,"FacialExpressionRecognition", InternationalJournalonComputerScienceandEngineering, Vol.02, No. 05, 2010,1552-1557.

[15] Frank Y. Shih, Chao-Fa Chuang, Patrick S. P. Wang "Performance Comparisons Of Facial Expression Recognition In Jaffe Database" International Journal of Pattern Recognition and Artificial Intelligence Vol. 22, No. 3 (2008)445-459. 